\title{
HIGH ORDER METHODS FOR A CLASS OF VOLTERRA INTEGRAL EQUATIONS WITH WEAKLY SINGULAR KERNELS*
}

\author{
FRANK DE HOOG† AND RICHARD WEISS $\ddagger$
}

Abstract. The solution of the Volterra integral equation

$$
x(t)=g_{1}(t)+\sqrt{t} g_{2}(t)+\int_{0}^{t} \frac{K(t, s, x(s))}{\sqrt{t-s}} d s, \quad 0 \leqq t \leqq T,
$$

where $g_{1}(t), g_{2}(t)$ and $K(t, s, x)$ are smooth functions, can be represented as $x(t)=u(t)+\sqrt{t} v(t)$, $0 \leqq t \leqq T$, where $u(t), v(t)$ are smooth and satisfy a system of Volterra integral equations. In this paper, numerical schemes for the solution of $\left(^{*}\right)$ are suggested which calculate $x(t)$ via $u(t), v(t)$ in a neighborhood of the origin and use $\left(^{*}\right)$ on the rest of the interval $0 \leqq t \leqq T$. In this way, methods of arbitrarily high order can be derived. As an example, schemes based on the product integration analogue of Simpson's rule are treated in detail. The schemes are shown to be convergent of order $h^{7 / 2}$. Asymptotic error estimates are derived in order to examine the numerical stability of the methods.

1. Introduction. The Volterra integral equation of the second kind

$$
x(t)=g_{1}(t)+\sqrt{t} g_{2}(t)+\int_{0}^{t} \frac{K(t, s, x(s))}{\sqrt{t-s}} d s, \quad 0 \leqq t \leqq T,
$$

arises in a number of problems in mathematical physics (see Chambre [1], Levinson [5] and Keller and Olmstead [4]).

It has been shown (see for instance Miller and Feldstein [9]) that (1.1) possesses a unique continuous solution on $[0, T]$ if

(i) $g_{1}(t)+\sqrt{t} g^{2}(t)$ is continuous on $0 \leqq t \leqq T$,

(ii) $K(t, s, x)$ is continuous with respect to $t$ and $s$ and uniformly Lipschitz continuous with respect to $x$ for all $x$.

It is clear that the accuracy of a numerical method for (1.1) willodepend on the smoothness of $K(t, s, x(s))$. The smoothness of $x(t)$ is considered in the following theorem which is a slight generalization of Theorem 3.2 in de Hoog and Weiss [2].

THEOREM 1.1. Let

(i) $g_{1}(t)$ and $g_{2}(t)$ be $n$ times continuously differentiable on $0 \leqq t \leqq T$,

(ii) $K(t, s, x)$ satisfy condition (ii) above and in addition be $n$ times continuously differentiable with respect to $t$ and $s$ and $2 n$ times continuously differentiable with respect to $x$ for $0 \leqq s \leqq t \leqq T$ and $x$ in an open neighborhood of $x(t)$.

Then

$$
x(t)=u(t)+\sqrt{t} v(t), \quad u(t), v(t) \in C^{n}[0, T]
$$

* Received by the editors December 4, 1972, and in revised form December 22, 1973.

$\uparrow$ Mathematics Department, University of California at Los Angeles, Los Angeles, California 90024. 91109.

$\ddagger$ Applied Mathematics Department, California Institute of Technology, Pasadena, California 
where $u(t), v(t)$ is the solution of the system

$$
\begin{array}{ll}
u(t)=g_{1}(t)+\int_{0}^{t} \frac{\sqrt{s}}{\sqrt{t-s}} K_{1}(t, s, u(s), v(s)) d s, & 0 \leqq t \leqq T, \\
v(t)=g_{2}(t)+\frac{1}{\sqrt{t}} \int_{0}^{t} \frac{K_{2}(t, s, u(s), v(s))}{\sqrt{t-s}} d s, & 0 \leqq t \leqq T,
\end{array}
$$

and

$$
\begin{aligned}
& K_{1}(t, s, u, v)=\frac{K(t, s, u+\sqrt{s} v)-K(t, s, u-\sqrt{s} v)}{2 \sqrt{s}} \\
& K_{2}(t, s, u, v)=\frac{K(t, s, u+\sqrt{s} v)+K(t, s, u-\sqrt{s} v)}{2} .
\end{aligned}
$$

Hence, in general $K(t, s, x(s))$ is not smooth in a neighborhood of the origin and so a finite difference scheme based on a piecewise polynomial approximation to $K(t, s, x(s))$ yields only a low order of convergence. On the other hand, $K_{1}(t, s, u(s), v(s))$ and $K_{2}(t, s, u(s), v(s))$ are smooth and this indicates that schemes for (1.2) may be more efficient.

However, it is important to note that in general the system (1.2) obtained from a stable equation (1.1) is not stable. This can be illustrated by the simple example,

$$
x(t)=1-\int_{0}^{t} \frac{x(s)}{\sqrt{t-s}} d s,
$$

which, by a Laplace transform argument, has the solution

$$
x(t)=\exp (\pi t) \operatorname{erfc}(\sqrt{\pi t}),
$$

where

$$
\operatorname{erfc}(t)=\frac{2}{\sqrt{\pi}} \int_{t}^{\infty} \exp \left(-s^{2}\right) d s .
$$

The solution for the system corresponding to (1.2) is

$$
u(t)=\exp (\pi t)
$$

and

$$
v(t)=\frac{\exp (\pi t)}{\sqrt{t}}\{\operatorname{erfc} \sqrt{\pi t}-1\} .
$$

Clearly, while $x(t)$ is bounded, $u(t)$ and $v(t)$ increase exponentially.

In this paper, we shall construct numerical schemes for (1.1) which utilize (1.2) only in a neighborhood of the origin and (1.1) on the rest of the interval. In this way it is possible to take advantage of the smoothness properties of (1.2) without letting the effect of the instability become dominant. The analysis of convergence and numerical stability of these methods represents the main part of the paper. 
2. Preliminaries. In the sequel, we shall use the notation

$$
\begin{gathered}
t_{i}=i h, \quad i=0, \cdots, I ; \quad h=T / I, \\
\omega(t)=\prod_{k=0}^{2}(t-k), \quad l_{k}(t)=\frac{\omega(t) H(t) H(2-t)}{(t-k) \omega^{\prime}(k)}, \quad k=0,1,2, \\
w(t)=\prod_{k=0}^{3}(t-k), \quad L_{k}(t)=\frac{w(t) H(t) H(3-t)}{(t-k) w^{\prime}(k)}, \quad k=0,1,2,3,
\end{gathered}
$$

where $H(t)=1$ for $t \geqq 0$ and $H(t)=0$ for $t<0$.

The following lemmas will be required in $\S \S 4$ and 5 .

LEMMA 2.1. There exists a constant $K$ such that

$$
\sum_{l=1}^{i-1} \frac{1}{l^{3 / 2}(i-l)^{1 / 2}} \leqq \frac{K}{(i-1)^{1 / 2}}, \quad i \geqq 2
$$

Proof.

$$
\begin{aligned}
\sum_{l=1}^{i-1} \frac{1}{l^{3 / 2}(i-l)^{1 / 2}} & \leqq \frac{1}{(i-1)^{1 / 2}}+\frac{1}{(i-1)^{3 / 2}}+\int_{1}^{i-2} \frac{1}{s^{3 / 2}(i-1-s)^{1 / 2}} d s \\
& \leqq \frac{3}{(i-1)^{1 / 2}} .
\end{aligned}
$$

LEMMA 2.2. If $f(t)$ is continuously differentiable, $F(t)=f(t)$ or $F(t)=\sqrt{t} f(t)$, $u=0$ or 1 and $0<s<1$, then

$$
h \sum_{l=0}^{i-1} \frac{F\left(t_{l}+s h\right)}{\sqrt{t_{i}+u h-t_{l}-s h}}=\int_{0}^{t_{i}} \frac{F(t)}{\sqrt{t_{i}-t}} d t+h^{1 / 2} F\left(t_{i}\right) \alpha(u, s)+O\left(h / \sqrt{t_{i}}\right),
$$

where

$$
\alpha(u, s)= \begin{cases}\tilde{\zeta}\left(\frac{1}{2}, 1-s\right), & u=0 \\ \tilde{\zeta}\left(\frac{1}{2}, 1-s\right)-\frac{1}{\sqrt{1-s}}, & u=1\end{cases}
$$

and $\tilde{\zeta}(s, a)$ is the generalized periodic zeta function.

Proof. The lemma follows immediately from the generalized Euler Maclaurin sum formula for integrands with algebraic singularities given in Lyness and Ninham [8].

The following result can be obtained if the hypothesis on $f(t)$ in Lemma 2.2 is weakened.

LEMMA 2.3. If $f(t)$ is Hölder continuous with exponent $\frac{1}{2}, u=0$ or $1,0<s<1$ and $F(t)=f(t)$ or $F(t)=\sqrt{t} f(t)$, then

$$
h \sum_{l=0}^{i-1} \frac{F\left(t_{l}+s h\right)}{\sqrt{t_{i}+u h-t_{l}-s h}}=\int_{0}^{t_{i}} \frac{F(t)}{\sqrt{t_{i}-t}} d t+O\left(h^{1 / 2} / \sqrt{1+u-s}\right) .
$$


Proof.

$$
\begin{aligned}
& \left|\sum_{l=0}^{i-1}\left\{h \frac{F\left(t_{l}+s h\right)}{\sqrt{t_{i}+u h-t_{l}-s h}}-\int_{t_{l}}^{t_{l}+1} \frac{F(t)}{\sqrt{t_{i}-t}} d t\right\}\right| \\
& \leqq h K \sum_{l=0}^{i-1}\left|\frac{1}{\sqrt{t_{i}+u h-t_{l}-s h}}-\frac{1}{\sqrt{t_{i}-t_{l}}}\right|+O\left(h^{1 / 2}\right)
\end{aligned}
$$

for some constant $K$. The result now follows from the generalized Euler-Maclaurin sum formula.

3. Numerical schemes. Linz [6] used product integration to obtain finite difference schemes for equations of the form

$$
x(t)=g(t)+\int_{0}^{t} K(t, s, x(s)) p(t, s) d s, \quad 0 \leqq t \leqq T,
$$

where $K(t, s, x(s))$ is "smooth" and $p(t, s)$ may have singularities (n.b. (3.1) may be a system of equations): By discretizing (3.1) at $t_{i}, i=1, \cdots, I$, and using the approximation

$$
\tilde{K}\left(t_{i}, s, x(s)\right)=\sum_{l=0}^{i} C_{i l}(s) K\left(t_{i}, t_{l}, x\left(t_{l}\right)\right), \quad i=q, \cdots, I,
$$

for $K\left(t_{i}, s, x(s)\right)$ on $0 \leqq s \leqq t_{i}$, product integration yields the scheme

$$
x_{i}=g\left(t_{i}\right)+\sum_{l=0}^{i} B_{i l} K\left(t_{i}, t_{l}, x_{l}\right), \quad i=q, \cdots, I,
$$

where

$$
B_{i l}=\int_{0}^{t_{i}} C_{i l}(s) p\left(t_{i}, s\right) d s
$$

and $x_{i}$ denotes the numerical approximation to $x\left(t_{i}\right)$.

The scheme (3.2) requires $q$ starting values $x_{0}, \cdots, x_{q-1}$ which must be determined independently. If $B_{i i} \neq 0$, the scheme is implicit and requires the solution of a nonlinear equation at each step.

The concept of a repetition factor as introduced by Linz [7] for second kind Volterra equations with "smooth" kernels (i.e., $p(t, s)=1$ ) can be generalized in the following way to include the scheme (3.2).

Definition. The scheme (3.2) has a repetition factor $p$ if $p$ is the smallest integer such that

$$
C_{i l}(t)=C_{i+p, l}(t), \quad 0 \leqq t \leqq t_{i},
$$

for $l=0,1, \cdots, i-r$, where $r$ is a fixed integer independent of $i$.

In the remainder of the paper we shall consider schemes which are obtained in the following way. If $i$ is even, $K\left(t_{i}, s, x(s)\right), i \geqq 2$, is approximated by a piecewise quadratic interpolating to $K\left(t_{i}, s, x(s)\right)$ at the points $t_{2 l}, t_{2 l+1}$ and $t_{2 l+2}$. If $i$ is odd this can be extended as follows.

(a) $K\left(t_{i}, s, x(s)\right)$ is approximated by a piecewise quadratic on $\left[0, t_{i-3}\right]$ and a cubic interpolating to $K\left(t_{i}, s, x(s)\right)$ at $t_{i-3}, t_{i-2}, t_{i-1}$ and $t_{i}$ on $\left[t_{i-3}, t_{i}\right]$, 
or

(b) on $[0,3 h], K\left(t_{i}, s, x(s)\right)$ is approximated by a cubic interpolating at $0, h, 2 h$ and $3 h$ and on $\left[3 h, t_{i}\right]$ by a piecewise quadratic interpolating at $t_{2 l+1}$, $t_{2 l+2}$ and $t_{2 l+3}$.

Then,

$$
\begin{aligned}
& \text { (a) } C_{2 i, 0}(s)=l_{0}\left(\frac{s}{h}\right) \text {, } \\
& C_{2 i, 2 l}(s)=l_{2}\left(\frac{s-t_{2 l-2}}{h}\right)+l_{0}\left(\frac{s-t_{2 l}}{h}\right), \quad l=1, \cdots, i-1, \\
& C_{2 i, 2 l+1}(s)=l_{1}\left(\frac{s-t_{2 l}}{h}\right), \quad l=0, \cdots, i-1, \\
& C_{2 i, 2 i}(s)=l_{2}\left(\frac{s-t_{2 i-2}}{h}\right) \text {, } \\
& C_{2 i+1, l}(s)=C_{2 i, l}(s) \text {, } \\
& l=0, \cdots, 2 i-3 \\
& C_{2 i+1,2 i-2}(s)=l_{2}\left(\frac{s-t_{2 i-4}}{h}\right)+L_{0}\left(\frac{s-t_{2 i-2}}{h}\right) \text {, } \\
& C_{2 i+1,2 i-2+r}(s)=L_{r}\left(\frac{s-t_{2 i-2}}{h}\right), \\
& r=1,2,3 \\
& \text { (b) } \hat{C}_{2 i, l}(s)=C_{2 i, l}(s) \text {, } \\
& \hat{C}_{2 i+1, r}(s)=L_{r}\left(\frac{s}{h}\right) \text {, } \\
& \hat{C}_{2 i+1,3}(s)=L_{3}\left(\frac{s}{h}\right)+l_{0}\left(\frac{s-3 h}{h}\right), \\
& \widehat{C}_{2 i+1,2 l}(s)=l_{1}\left(\frac{s-t_{2 l-1}}{h}\right) \text {, } \\
& l=2, \cdots, i, \\
& \hat{C}_{2 i+1,2 l+1}(s)=l_{2}\left(\frac{s-t_{2 l-1}}{h}\right)+l_{0}\left(\frac{s-t_{2 l+1}}{h}\right), \quad l=2, \cdots, i-1, \\
& \widehat{C}_{2 i+1,2 i+1}=l_{2}\left(\frac{s-t_{2 i-1}}{h}\right) \text {. }
\end{aligned}
$$

The methods (a) and (b) have a repetition factor of one and two respectively and can be thought of as generalizations of the schemes Simpson\#1 and \#2 for Volterra integral equations of the second kind with "smooth" kernels investigated by Linz [7] and Noble [10]. Clearly the above can be generalized to piecewise polynomial interpolation of higher order. However the present schemes contain most of the features of the more general class and the analysis given in the sequel generalizes easily. A similar analysis can also be used to obtain corresponding results for the block-by-block methods suggested by Linz [6].

As suggested in $\S 1$, the above schemes will be applied in the following way. 
On $0 \leqq t \leqq a, a>0$, approximations to $u(t)$ and $v(t)$ are obtained via (1.2). For $a \leqq t \leqq T,(1.1)$ can be rewritten as

$$
x(t+a)=G(t+a)+\int_{0}^{t} \frac{K(t+a, s+a, x(s+a))}{\sqrt{t-s}} d s, \quad 0 \leqq t \leqq T-a,
$$

where

$$
G(t+a)=g_{1}(t+a)+\sqrt{t+a} g_{2}(t+a)+\int_{0}^{a} \frac{K(t+a, s, x(s))}{\sqrt{t+a-s}} d s .
$$

The term $G(t+a)$ is approximated by applying product integration to each of the terms on the right-hand side of

$$
\begin{aligned}
\int_{0}^{a} \frac{K(t+a, s, y(s))}{\sqrt{t+a-s}} d s= & \int_{0}^{a} \sqrt{s} \frac{K_{1}(t+a, s, u(s), v(s))}{\sqrt{t+a-s}} d s \\
& +\int_{0}^{a} \frac{K_{2}(t+a, s, u(s), v(s))}{\sqrt{t+a-s}} d s .
\end{aligned}
$$

Using this approximation for $G(t+a)$, a scheme applied to (3.3) is then used for the calculation of $x(t)$ on $a \leqq t \leqq T$.

For the analysis of these composite methods it is sufficient to investigate the schemes for (1.2) and (3.3) separately. Although (3.3) has a "smooth" solution, it is of the form (1.1). Hence, for notational convenience, we shall consider the schemes applied to (1.1) rather than (3.3).

The finite difference methods for (1.1) corresponding to (a) and (b) are

$$
x_{i}=g_{1}\left(t_{i}\right)+\sqrt{t_{i}} g_{2}\left(t_{i}\right)+\sum_{l=0}^{i} W_{i l} K\left(t_{i}, t_{l}, x_{l}\right), \quad i=2, \cdots, I,
$$

and

$$
\hat{x}_{i}=g_{1}\left(t_{i}\right)+\sqrt{t}_{i} g_{2}\left(t_{i}\right)+\sum_{l=0}^{i} \hat{W}_{i l} K\left(t_{i}, t_{l}, \hat{x}_{l}\right), \quad i=2, \cdots, I,
$$

where

$$
W_{i l}=\int_{0}^{t_{i}} \frac{C_{i l}(s)}{\sqrt{t_{i}-s}} d s, \quad \hat{W}_{i l}=\int_{0}^{t_{i}} \frac{\hat{C}_{i l}(s)}{\sqrt{t_{i}-s}} d s,
$$

and $x_{i}, \hat{x}_{i}$ are the approximations to $x\left(t_{i}\right)$.

Similarly, the scheme for (1.2) using method (a) is

$$
\begin{array}{ll}
u_{i}=g_{1}\left(t_{i}\right)+\sum_{l=0}^{i} Y_{i l} K_{1}\left(t_{i}, t_{l}, u_{l}, v_{l}\right), & i=2, \cdots, I, \\
v_{i}=g_{2}\left(t_{i}\right)+\frac{1}{\sqrt{t_{i}}} \sum_{l=0}^{i} W_{i l} K_{2}\left(t_{i}, t_{l}, u_{l}, v_{l}\right), & i=2, \cdots, I,
\end{array}
$$

where

$$
Y_{i l}=\int_{0}^{t_{i}} \frac{\sqrt{s} C_{i l}(s)}{\sqrt{t_{i}-s}} d s
$$


and the $W_{i l}$ are as above. The corresponding scheme for method (b), subsequently denoted as (3.6b), is obtained by replacing $u_{i}, v_{i}, Y_{i l}, W_{i l}$ in (3.6a) by $\hat{u}_{i}, \hat{v}_{i}, \hat{Y}_{i l}, \hat{W}_{i i}^{\prime}$.

It follows from contraction mapping arguments that $(3.5 \mathrm{a}, \mathrm{b})$ and $(3.6 \mathrm{a}, \mathrm{b})$ are uniquely solvable if $h$ is sufficiently small.

4. Convergence. In the sequel, we shall assume that $K(t, s, x(s)), K_{1}(t, s, u(s)$, $v(s))$ and $K_{2}(t, s, u(s), v(s))$ are sufficiently smooth. Subtracting (1.1) from (3.5a), (1.2) from (3.6a) and defining

$$
\alpha_{i}=x_{i}-x\left(t_{i}\right), \quad \beta_{i}=u_{i}-u\left(t_{i}\right), \quad \gamma_{i}=\left(v_{i}-v\left(t_{i}\right)\right) \sqrt{t_{i}},
$$

we obtain

$$
\begin{array}{ll}
\alpha_{i}=\sum_{l=0}^{i} W_{i l}\left(K\left(t_{i}, t_{l}, x_{l}\right)-K\left(t_{i}, t_{l}, x\left(t_{l}\right)\right)+P_{i},\right. & i=2, \cdots, I, \\
\beta_{i}=\sum_{l=0}^{i} Y_{i l}\left(K_{1}\left(t_{i}, t_{l}, u_{l}, v_{l}\right)-K_{1}\left(t_{i}, t_{l}, u\left(t_{l}\right), v\left(t_{l}\right)\right)+Q_{i},\right. & i=2, \cdots, I, \\
\gamma_{i}=\sum_{l=0}^{i} W_{i l}\left(K_{2}\left(t_{i}, t_{l}, u_{l}, v_{l}\right)-K_{2}\left(t_{i}, t_{l}, u\left(t_{l}\right), v\left(t_{l}\right)\right)+R_{i},\right. & i=2, \cdots, I,
\end{array}
$$

where

$$
\begin{aligned}
P_{i} & =\sum_{l=0}^{i} W_{i l} K\left(t_{i}, t_{l}, x\left(t_{l}\right)\right)-\int_{0}^{t_{i}} \frac{K\left(t_{i}, s, x(s)\right)}{\sqrt{t_{i}-s}} d s, \\
Q_{i} & =\sum_{l=0}^{i} Y_{i l} K_{1}\left(t_{i}, t_{l}, u\left(t_{l}\right), v\left(t_{l}\right)\right)-\int_{0}^{t_{i}} \frac{\sqrt{s}}{\sqrt{t_{i}-s}} K_{1}\left(t_{i}, s, u(s), v(s)\right) d s \\
R_{i} & =\sum_{l=0}^{i} W_{i l} K_{2}\left(t_{i}, t_{l}, u\left(t_{l}\right), v\left(t_{l}\right)\right)-\int_{0}^{t_{i}} \frac{K_{2}\left(t_{i}, s, u(s), v(s)\right)}{\sqrt{t_{i}-s}} d s .
\end{aligned}
$$

Corresponding equations for (3.5b) and (3.6b) are obtained by replacing $\alpha_{i}, W_{i, l}$, $P_{i}, \cdots$ by $\tilde{\alpha}_{i}, \hat{W}_{i, l}, \hat{P}_{i}, \cdots$.

The following lemma examines the asymptotic behavior of $P_{i}, Q_{i}, R_{i}$ and $\hat{P}_{i}, \hat{Q}_{i}, \hat{R}_{i}$.

LEMMA 4.1. There exist continuously differentiable functions $\phi_{r}(t), \psi_{r}(t)$ and $\theta_{r}(t), r=0,1$, such that for $i \geqq 1$,

$$
\begin{aligned}
& P_{2 i+r}=h^{7 / 2} \phi_{r}\left(t_{2 i+r}\right)+O\left(\frac{h^{4}}{\sqrt{t_{2 i+r}}}\right) \\
& Q_{2 i+r}=h^{7 / 2} \sqrt{t_{2 i+r}} \psi_{r}\left(t_{2 i+r}\right)+O\left(\frac{h^{4}}{\sqrt{t_{2 i+r}}}\right) \\
& R_{2 i+r}=h^{7 / 2} \theta_{r}\left(t_{2 i+r}\right)+O\left(\frac{h^{4}}{\sqrt{t_{2 i+r}}}\right)
\end{aligned}
$$

Corresponding relations for $\hat{P}_{2 i+r}, \hat{Q}_{2 i+r}$ and $\hat{R}_{2 i+r}, r=0,1$, with $\phi_{r}(t), \psi_{r}(t)$ and $\theta_{r}(t)$ replaced by $\hat{\phi}_{r}(t), \hat{\psi}_{r}(t)$ and $\hat{\theta}_{r}(t)$ respectively are also valid. 
Proof. Let $K(t, s)=K(t, s, x(s))$. By definition,

$$
\sum_{l=0}^{2 i} W_{2 i, l} K\left(t_{2 i}, t_{l}\right)=\sum_{l=0}^{i-1} \sum_{k=0}^{2} K\left(t_{2 i}, t_{2 l+k}\right) \int_{t_{2 l}}^{t_{2 l+2}} \frac{l_{k}\left(\left(s-t_{2 l}\right) / h\right)}{\sqrt{t_{2 i}-s}} d s .
$$

From de Hoog and Weiss [3, Lemma 2.1],

$$
\begin{aligned}
& \sum_{l=0}^{i-1} \sum_{k=0}^{2} K\left(t_{2 i}, t_{2 l+k}\right) \int_{t_{2 l}}^{t_{2 l+2}} \frac{l_{k}\left(\left(s-t_{2 l}\right) / h\right)}{\sqrt{t_{2 i}-s}} d s-\int_{0}^{t_{2 i}} \frac{K\left(t_{2 i}, s\right)}{\sqrt{t_{2 i}-s}} d s \\
&=\text { const. } h^{3} \int_{0}^{1}\left\{\omega(2 s) 2 h \sum_{l=0}^{i-1} \frac{K^{(3)}\left(t_{2 i}, t_{2 l}+2 s h\right)}{\sqrt{t_{2 i}-t_{2 l}-2 s h}}\right\} d s+O\left(h^{4}\right),
\end{aligned}
$$

where $K^{(q)}(t, s)=\partial^{q} K(t, s) / \partial s^{q}$. The result for $P_{2 i}$ now follows from Lemma 2.2 with $u=0$ and the fact that $\int_{0}^{1} \omega(2 s) d s=0$.

To obtain the asymptotic estimate for $P_{2 i+1}$, note that from Lagrangian interpolation,

$$
\begin{aligned}
\sum_{l=0}^{2 i+1} W_{2 i+1, l} K\left(t_{2 i+1}, t_{l}\right)= & \sum_{l=0}^{i-2} \sum_{k=0}^{2} K\left(t_{2 i+1}, t_{2 l+k}\right) \int_{t_{2 l}}^{t_{2 l+2}} \frac{l_{k}\left(\left(s-t_{2 l}\right) / h\right)}{\sqrt{t_{2 i+1}-s}} d s \\
& +\int_{t_{2 i-2}}^{t_{2 i+1}} \frac{K\left(t_{2 i+1}, s\right)}{\sqrt{t_{2 i+1}-s}} d s+O\left(h^{4}\right) .
\end{aligned}
$$

Proceeding in the same way as for $P_{2 i}$, but using Lemma 2.2 with $u=1$, yields the result. The rest of the lemma follows in a similar way.

We shall now prove the following convergence result for the schemes $(3.5 \mathrm{a}, \mathrm{b})$.

THEOREM 4.1. Let

$$
x_{r}-x\left(t_{r}\right)=\delta_{r}, \quad \hat{x}_{r}-x\left(t_{r}\right)=\hat{\delta}_{r}, \quad \delta_{r}=O(\delta), \hat{\delta}_{r}=O(\hat{\delta}), \quad r=0,1 .
$$

Then there exist constants $K, \hat{K}$ such that

$$
\begin{array}{ll}
\left|\alpha_{i}\right| \leqq K\left(h^{7 / 2}+\frac{h \delta}{\sqrt{t}}\right), & i=2, \cdots, I, \\
\left|\hat{\alpha}_{i}\right| \leqq \hat{K}\left(h^{7 / 2}+\frac{h \hat{\delta}}{\sqrt{t_{i}}}\right), & i=2, \cdots, I .
\end{array}
$$

Proof. Since the arguments for the scheme (3.5a) can be extended to (3.5b), only the scheme (3.5a) will be considered. Let $\tilde{x}_{i}, i=2, \cdots, I$, be the solution of (3.5a) obtained with exact starting values, i.e.,

$$
\begin{array}{rlrl}
\tilde{x}_{i}=g_{1}\left(t_{i}\right) & +\sqrt{t_{i}} g_{2}\left(t_{i}\right)+W_{i, 0} K\left(t_{i}, 0, x(0)\right)+W_{i, 1} K\left(t_{i}, h, x(h)\right) \\
& +\sum_{l=2}^{i} W_{i, l} K\left(t_{i}, t_{l}, \tilde{x}_{l}\right), & i=2, \cdots, I .
\end{array}
$$

Clearly,

$$
\alpha_{i}=\left(x_{i}-\tilde{x}_{i}\right)+\left(\tilde{x}_{i}-x\left(t_{i}\right)\right)
$$


We now estimate the terms in brackets separately. Subtraction of (3.5a) from (4.3) yields

$$
\begin{aligned}
\tilde{x}_{i}-x_{i}= & W_{i, 0}\left(K\left(t_{i}, 0, x(0)\right)-K\left(t_{i}, 0, x_{0}\right)\right)+W_{i, 1}\left(K\left(t_{i}, h, x(h)\right)\right. \\
\left.-K\left(t_{i}, h, x_{1}\right)\right)+\sum_{l=2}^{i} W_{i, l}\left(K\left(t_{i}, t_{l}, \tilde{x}_{l}\right)-K\left(t_{i}, t_{l}, x_{l}\right)\right), & \\
& i=2, \cdots, I .
\end{aligned}
$$

Let $C$ be a constant such that

$$
\begin{aligned}
\left|K\left(t_{i}, r h, x(r h)\right)-K\left(t_{i}, r h, x_{r}\right)\right| \leqq \sqrt{C} \delta, & r=0,1, & i=2, \cdots, I, \\
\left|W_{i, r}\right| \leqq \sqrt{C} h / \sqrt{t_{i}}, & r=0,1, & i=2, \cdots, I,
\end{aligned}
$$

and define

$$
\tilde{\alpha}_{i}=\left|\left(\tilde{x}_{i}-x_{i}\right) \sqrt{t_{i}}\right|, \quad i=2, \cdots, I .
$$

Multiplying (4.5) by $\sqrt{t}_{i}$, taking absolute values and applying the Lipschitz inequality for $K(t, s, x)$ yields

$$
\tilde{\alpha}_{i} \leqq \sqrt{t_{i}} L \sum_{l=2}^{i} \frac{\left|W_{i, l}\right|}{\sqrt{t_{l}}} \tilde{\alpha}_{l}+C h \delta, \quad i=2, \cdots, I .
$$

Since $\sum_{l=2}^{i}\left|W_{i, l}\right| / \sqrt{t_{l}}, i=2, \cdots, I$, are uniformly bounded, it follows in the same way as in $\operatorname{Linz}[6]$ that

$$
\tilde{\alpha}_{i} \leqq K_{1} h \delta, \quad i=2, \cdots, I .
$$

To estimate $\left|\tilde{x}_{i}-x\left(t_{i}\right)\right|$, we examine (4.1) with $x_{r}=x\left(t_{r}\right), r=0,1$. Using the Lipschitz continuity of $K(t, s, x)$ and Lemma 4.1 , we obtain

$$
\left|\tilde{x}_{i}-x\left(t_{i}\right)\right| \leqq L \sum_{l=2}^{i}\left|W_{i, l}\right|\left|\tilde{x}_{l}-x\left(t_{l}\right)\right|+O\left(h^{7 / 2}\right), \quad i=2, \cdots, I .
$$

Hence, using the arguments in Linz [6], we obtain

$$
\left|\tilde{x}_{i}-x\left(t_{i}\right)\right| \leqq K_{2} h^{7 / 2}, \quad i=2, \cdots, I .
$$

The result follows.

The following convergence result for the scheme (3.6a) is obtained in a similar way. An analogous result holds for scheme (3.6b).

THEOREM 4.2. Let

$$
u_{r}-u\left(t_{r}\right)=\delta_{r}, \quad v_{r}-v\left(t_{r}\right)=\eta_{r} ; \quad \delta_{r}, \eta_{r}=O(\sigma), \quad r=0,1 .
$$

Then there exists a constant $K$ such that

$$
\left|\beta_{i}\right|,\left|\gamma_{i}\right| \leqq K\left(h^{7 / 2}+\sigma h / \sqrt{t_{i}}\right), \quad i=2, \cdots, I,
$$

5. Asymptotic expansions and numerical stability. In this section we shall derive asymptotic error estimates for the schemes $(3.5 \mathrm{a}, \mathrm{b})$. Firstly we consider (3.5a). In order to study the effect of rounding errors, we assume that starting errors $x_{r}-x\left(t_{r}\right)=\delta_{r}, \delta_{r}=O(\delta), r=0,1$, are present. To simplify the notation, 
we introduce

$$
k(t, s)=\frac{\partial K}{\partial x}(t, s, x(s))
$$

and

$$
f_{r}(t)= \begin{cases}\frac{K\left(t, 0, x_{r}\right)-K\left(t, 0, x\left(t_{r}\right)\right)}{x_{r}-x\left(t_{r}\right)}, & x_{r} \neq x\left(t_{r}\right), \quad r=0,1, \\ \frac{\partial K}{\partial x}\left(t, 0, x\left(t_{r}\right)\right), & x_{r}=x\left(t_{r}\right), \quad r=0,1 .\end{cases}
$$

For simplicity of notation, we shall assume that $I$ is even.

THEOREM 5.1. Let $\zeta_{r}(t), r=0,1$, be the solution of the system

$$
\zeta_{r}(t)=\phi_{r}(t)+\int_{0}^{t} \frac{k(t, s)}{\sqrt{t-s}}\left(\frac{1}{3} \zeta_{0}(s)+\frac{2}{3} \zeta_{1}(s)\right) d s, \quad r=0,1,
$$

and let $y(t)$ be the solution of

$$
y(t)=\delta_{0} F_{0}(t)+\delta_{1} F_{1}(t)+\int_{0}^{t} \frac{k(t, s)}{\sqrt{t-s}} y(s) d s,
$$

where

$$
F_{r}(t)=a_{r} \int_{0}^{t} \frac{k(t, s) f_{r}(s)}{\sqrt{s} \sqrt{t-s}} d s
$$

and $a_{0}=1 / 3, a_{1}=4 / 3$. Then

$$
\begin{aligned}
\alpha_{2 i+r}= & h^{7 / 2} \zeta_{r}\left(t_{2 i+r}\right)+\delta_{0} f_{0}\left(t_{2 i+r}\right) W_{2 i+r, 0}+\delta_{1} f_{1}\left(t_{2 i+r}\right) W_{2 i+r, 1} \\
& +h y\left(t_{2 i+r}\right)+O\left(\frac{h^{4}}{\sqrt{t_{2 i+r}}}\right)+O\left(\frac{h^{3 / 2} \delta}{\sqrt{t_{2 i+r}}}\right), \quad r=0,1, \quad i=1, \cdots, I / 2 .
\end{aligned}
$$

Proof. Applying Taylor's theorem to (4.1) and using Theorem 4.1 and Lemma 4.1, we obtain

$$
\begin{aligned}
\alpha_{2 i+r}= & \sum_{l=2}^{2 i+r} W_{2 i+r, l} k\left(t_{2 i+r}, t_{l}\right) \alpha_{l}+h^{7 / 2} \phi_{r}\left(t_{2 i+r}\right) \\
& +W_{2 i+r, 0} \delta_{0} f_{0}\left(t_{2 i+r}\right)+W_{2 i+r, 1} \delta_{1} f_{1}\left(t_{2 i+r}\right) \\
& +O\left(\frac{h^{4}}{\sqrt{t_{2 i+r}}}\right)+O\left(\frac{h^{3 / 2} \delta}{\sqrt{t_{2 i+r}}}\right), \quad r=0,1, \quad i=1, \cdots, I / 2 .
\end{aligned}
$$

Clearly, $\alpha_{i}=p_{i}+q_{i}, i=2, \cdots, I$, where

$$
\begin{aligned}
p_{2 i+r}=\sum_{l=2}^{2 i+r} W_{2 i+r, l} k\left(t_{2 i+r}, t_{l}\right) p_{l}+h^{7 / 2} \phi_{r}\left(t_{2 i+r}\right) & +O\left(\frac{h^{4}}{\sqrt{t_{2 i+r}}}\right), \\
r & =0,1, \quad i=1, \cdots, I / 2,
\end{aligned}
$$


and

$$
\begin{aligned}
& q_{2 i+r}= \sum_{l=2}^{2 i+r} W_{2 i+r, l} k\left(t_{2 i+r}, t_{l}\right) q_{l}+W_{2 i+r, 0} \delta_{0} f_{0}\left(t_{2 i+r}\right) \\
&+W_{2 i+r, 1} \delta_{1} f_{1}\left(t_{2 i+r}\right)+O\left(\frac{h^{3 / 2} \delta}{\sqrt{t_{2 i+r}}}\right), \\
& r=0,1, \quad i=1, \cdots, I / 2 .
\end{aligned}
$$

Let

$$
q_{i}=W_{i, 0} \delta_{0} f_{0}\left(t_{i}\right)+W_{i, 1} \delta_{1} f_{1}\left(t_{i}\right)+s_{i}, \quad i=2, \cdots, I,
$$

Substitution into (5.5) yields

$$
\begin{aligned}
s_{i}= & \sum_{l=2 .}^{i} W_{i, l} k\left(t_{i}, t_{l}\right) s_{l} \\
& +\sum_{l=2}^{i} W_{i, l} k\left(t_{i}, t_{l}\right)\left(\delta_{0} W_{l, 0} f_{0}\left(t_{l}\right)+\delta_{1} W_{l, 1} f_{1}\left(t_{l}\right)\right)+O\left(\frac{h^{3 / 2} \delta}{\sqrt{t_{i}}}\right), \\
i & =2, \cdots, I .
\end{aligned}
$$

Clearly,

$$
\begin{aligned}
& \sum_{l=2}^{2 i+r} W_{2 i+r, l} k\left(t_{2 i+r}, t_{l}\right) W_{l, 0} f_{0}\left(t_{l}\right) \\
& =\int_{0}^{1}\left\{2 h \sum_{l=1}^{i-1} \frac{1}{\sqrt{t_{2 i+r}-t_{2 l}-2 s h}} \sum_{k=0}^{2} l_{k}(2 s) k\left(t_{2 i+r}, t_{2 l+k}\right) f_{0}\left(t_{2 l+k}\right) W_{2 l+k, 0}\right\} d s \\
& \quad+O\left(h^{3 / 2} / \sqrt{t_{2 i+r}}\right), \quad i=1, \cdots, I / 2 .
\end{aligned}
$$

Since

$$
\begin{aligned}
W_{2 l+k, 0} & =\int_{0}^{2 h} \frac{l_{0}(s / h)}{\left(t_{2 l+k}-s\right)^{1 / 2}} d s \\
(5.7) \quad & =\int_{0}^{2 h} \frac{l_{0}(s / h)}{\left(t_{2 l}+2 h \xi\right)^{1 / 2}} d s+\int_{0}^{2 h} l_{0}\left(\frac{s}{h}\right)\left(\frac{1}{\left(t_{2 l+k}-s\right)^{1 / 2}}-\frac{1}{\left(t_{2 l}+2 h \xi\right)^{1 / 2}}\right) d s \\
& =\frac{1}{\left(t_{2 l}+2 h \xi\right)^{1 / 2}} \int_{0}^{2 h} l_{0}\left(\frac{s}{h}\right) d s+O\left(\frac{h^{1 / 2}}{l^{3 / 2}}\right), \quad 0 \leqq \xi \leqq 1, \quad l \geqq 1,
\end{aligned}
$$

it follows that

$$
\begin{aligned}
& \sum_{l=2}^{2 i+r} W_{2 i+r, l} k\left(t_{2 i+r}, t_{l}\right) W_{l, 0} f_{0}\left(t_{l}\right) \\
& (5.8) \quad=\int_{0}^{2 h} l_{0}\left(\frac{s}{h}\right) d s \int_{0}^{1} 2 h \sum_{l=1}^{i-1}\left\{\frac{1}{\sqrt{t_{2 i+r}-t_{2 l}-2 s h}} \frac{1}{\sqrt{t_{2 l}+2 s h}}\right. \\
& \left.\cdot \sum_{k=0}^{2} \cdot l_{k}(2 s) k\left(t_{2 i+r}, t_{2 l+k}\right) f_{0}\left(t_{2 l+k}\right)\right\} d s+O\left(\sum_{l=1}^{i-1} \frac{h}{(i-l)^{1 / 2} l^{3 / 2}}\right)+O\left(\frac{h^{3 / 2}}{\sqrt{t_{2 i+r}}}\right), \\
& r=0,1, \quad i=1, \cdots, I / 2 .
\end{aligned}
$$


Applying the Euler-Maclaurin sum formula for integrands with algebraic singularities and Lemma 2.1 to the right-hand side of (5.8), we obtain

$$
\sum_{l=2}^{i} W_{i, l} k\left(t_{i}, t_{l}\right) W_{l, 0} f_{0}\left(t_{l}\right)=h F_{0}\left(t_{i}\right)+o\left(\frac{h^{3 / 2}}{\sqrt{t_{i}}}\right), \quad i=2, \cdots, I .
$$

Similarly,

$$
\sum_{l=2}^{i} W_{i, l} k\left(t_{i}, t_{l}\right) W_{l, 1} f_{1}\left(t_{l}\right)=h F_{1}\left(t_{i}\right)+O\left(\frac{h^{3 / 2}}{\sqrt{t}}\right), \quad i=2, \cdots, I .
$$

From Theorem 1.1,

$$
|y(t+h)-y(t)|=O\left(h^{1 / 2}\right), \quad 0 \leqq t \leqq T-h .
$$

Hence, using Lemma 2.3, we obtain

$$
\begin{aligned}
& \sum_{l=2}^{2 i+r} W_{2 i+r, l} k\left(t_{2 i+r}, t_{l}\right) y\left(t_{l}\right) \\
& =\int_{0}^{1}\left\{2 h \sum_{l=1}^{i-1} \frac{1}{\sqrt{t_{2 i+r}-t_{2 l}-2 s h}} \sum_{k=0}^{2} l_{k}(s) k\left(t_{2 i+r}, t_{2 l+k}\right) y\left(t_{2 l+k}\right)\right\} d s+O\left(h^{1 / 2}\right) \\
& =\int_{0}^{t_{2 i+r}} \frac{k\left(t_{2 i+r}, s\right) y(s)}{\sqrt{t_{2 i+r}-s}} d s+O\left(h^{1 / 2}\right), \quad r=0,1, \quad i=1, \cdots, I / 2 .
\end{aligned}
$$

Let $\tau_{i}=s_{i}-h y\left(t_{i}\right), i=2, \cdots, I$. Then subtraction of (5.11) from (5.6) and the use of (5.9) and (5.10) yield

$$
\tau_{i}=\sum_{l=2}^{i} W_{i l} k\left(t_{i}, t_{l}\right) \tau_{l}+o\left(\frac{h^{3 / 2} \delta}{\sqrt{t_{i}}}\right), \quad \quad i=2, \cdots, I .
$$

Hence, in the same way as in Theorem 4.1,

$$
\tau_{i}=o\left(\frac{h^{3 / 2} \delta}{\sqrt{t_{i}}}\right), \quad i=2, \cdots, I .
$$

To estimate $p_{i},(5.4)$ is rewritten as

$$
\begin{aligned}
p_{2 i+r}= & \sum_{l=1}^{i-1}\left(W_{2 i+r, 2 l} k\left(t_{2 i+r}, t_{2 l}\right) p_{2 l}+W_{2 i+r, 2 l+1} k\left(t_{2 i+r}, t_{2 l+1}\right) p_{2 l+1}\right) \\
& +h^{7 / 2} \phi_{r}\left(t_{2 i+r}\right)+O\left(\frac{h^{4}}{\sqrt{t_{2 i+r}}}\right), \quad r=0,1, \quad i=1, \cdots, I / 2 .
\end{aligned}
$$

In a similar way to $(5.7)$,

$$
W_{2 i+r, 2 l+k}=\frac{2 h b_{k}}{\left(t_{2 i+r}-t_{2 l}-2 h \xi\right)^{1 / 2}}+O\left(\frac{h^{1 / 2}}{(i-l)^{3 / 2}}\right), \quad 0 \leqq \xi \leqq 1,
$$

where $b_{0}=1 / 3, b_{1}=2 / 3$. Hence, a similar analysis to that used for the estimation of $s_{i}$ yields

$$
p_{2 i+r}=h^{7 / 2} \zeta_{r}\left(t_{2 i+r}\right)+O\left(\frac{h^{4}}{\sqrt{t_{2 i+r}}}\right), \quad r=0,1, \quad i=1, \cdots, I / 2 .
$$


In the following theorem, an asymptotic error estimate for the scheme $(3.5 b)$ is given.

THEOREM 5.2. Let $\hat{f}_{r}(t)$ be defined analogously to (5.1). Let $\hat{\zeta}_{r}(t), r=0,1$, be the solution of

$$
\hat{\zeta}_{r}(t)=\hat{\phi}_{r}(t)+\int_{0}^{t} \frac{k(t, s)}{\sqrt{t-s}}\left(a_{r} \hat{\zeta}_{0}(s)+b_{r} \hat{\zeta}_{1}(s)\right) d s, \quad r=0,1,
$$

and $\hat{y}_{r}(t), r=0,1$, be the solution of

$$
\hat{y}_{r}(t)=\hat{\delta}_{0} c_{r} \hat{F}_{0}(t)+\hat{\delta}_{1} d_{r} \hat{F}_{1}(t)+\int_{0}^{t} \frac{k(t, s)}{\sqrt{t-s}}\left(a_{r} \hat{y}_{0}(s)+b_{r} \hat{y}_{1}(s)\right) d s, \quad r=0,1,
$$

where

$$
\hat{F}_{r}(t)=\int_{0}^{t} \frac{k(t, s) \hat{f}_{r}(s)}{\sqrt{s} \sqrt{t-s}} d s, \quad r=0,1,
$$

and $a_{0}=b_{1}=\frac{1}{3}, a_{1}=b_{0}=\frac{2}{3}, c_{0}=\frac{26}{77}, c_{1}=\frac{25}{72}, d_{0}=\frac{86}{72}, d_{1}=\frac{91}{72}$. Then

$$
\begin{aligned}
\hat{\alpha}_{2 i+r}= & h^{7 / 2} \hat{\zeta}_{r}\left(t_{2 i+r}\right)+\hat{\delta}_{0} \hat{f}_{0}\left(t_{2 i+r}\right) \hat{W}_{2 i+r, 0}+\hat{\delta}_{1} f_{1}\left(t_{2 i+r}\right) \hat{W}_{2 i+r, 1} \\
& +h \hat{y}_{r}\left(t_{2 i+r}\right)+O\left(\frac{h^{4}}{\sqrt{t_{2 i+r}}}\right)+O\left(\frac{h^{3 / 2} \hat{\delta}}{\sqrt{t_{2 i+r}}}\right), \quad r=0,1, \quad i=1, \cdots, I / 2 .
\end{aligned}
$$

Proof. The proof proceeds as for Theorem 5.1. The only difference is that the error due to the inexact starting values no longer varies continuously with $t_{i}$ since the method has a repetition factor of two.

From (5.2),

$$
\begin{aligned}
& \zeta_{0}(t)-\zeta_{1}(t)=\phi_{0}(t)-\phi_{1}(t), \\
& \frac{1}{3} \zeta_{0}(t)+\frac{2}{3} \zeta_{1}(t)=\lambda(t),
\end{aligned}
$$

where

$$
\lambda(t)=\frac{1}{3} \phi_{0}(t)+\frac{2}{3} \phi_{1}(t)+\int_{0}^{t} \frac{k(t, s)}{\sqrt{t-s}} \lambda(s) d s .
$$

Hence $\zeta_{r}(t), r=0,1$, and $y(t)$ are obtained as the solutions of equations which possess the same kernel as the linearized equation of (1.1). Thus the arguments of Noble [10] imply that the scheme (3.5a) is numerically stable.

From (5.12),

$$
\begin{aligned}
& \hat{\zeta}_{0}(t)+\hat{\zeta}_{1}(t)=\hat{\lambda}_{1}(t), \\
& \hat{\zeta}_{0}(t)-\hat{\zeta}_{1}(t)=\hat{\lambda}_{2}(t),
\end{aligned}
$$

where

$$
\begin{aligned}
& \hat{\lambda}_{1}(t)=\hat{\phi}_{0}(t)+\hat{\phi}_{1}(t)+\int_{0}^{t} \frac{k(t, s)}{\sqrt{t-s}} \hat{\lambda}_{1}(s) d s, \\
& \hat{\lambda}_{2}(t)=\hat{\phi}_{0}(t)-\hat{\phi}_{1}(t)-\frac{1}{3} \int_{0}^{t} \frac{k(t, s)}{\sqrt{t-s}} \hat{\lambda}_{2}(s) d s .
\end{aligned}
$$


Hence, $\hat{\zeta}_{r}(t), r=0,1$ (and, clearly, also $\left.\hat{y}_{r}(t), r=0,1\right)$, may have unstable growth for a stable equation (1.1). For instance, if $k(t, s)=-1$, it follows from a Laplace transform argument that $\hat{\lambda}_{2}(t)$ behaves like $\exp (\sqrt{\pi / 3} t)$. Thus the scheme $(3.5 b)$ is not numerically stable.

It is clear that the preceding arguments can be generalized to show that all methods based on piecewise polynomial interpolation and having a repetition factor of one are numerically stable. On the other hand, numerical instability may occur in methods with a repetition factor greater than one. For Volterra integral equations of the second kind with smooth kernels, this result has been established by Linz [7] and Noble [10].

Asymptotic error estimates similar to those given in Theorems 5.1 and 5.2, can be obtained for the schemes $(3.6 \mathrm{a}, \mathrm{b})$. However, since the system (1.2) is unstable, for practical purposes the schemes $(3.6 \mathrm{a}, \mathrm{b})$ cannot be regarded as being numerically stable with respect to equation (1.1). It is therefore necessary to terminate the schemes $(3.6 \mathrm{a}, \mathrm{b})$ before the instability dominates.

6. Numerical example. The investigation of the radiation of heat from a semi-infinite solid having a constant heat source leads to the equation (see Keller and Olmstead [4])

$$
x(t)=\frac{1}{\sqrt{\pi}} \int_{0}^{t} \frac{1-x^{4}(s)}{\sqrt{t-s}} d s .
$$

The composite scheme (3.6a), (3.5a) was applied to this problem. The first pair of starting values for (3.6a) was obtained from (1.2), i.e.,

$$
u_{0}=u(0)=g_{1}(0), \quad v_{0}=v(0)=g_{2}(0)+2 K(0,0, u(0)) .
$$

The second pair, $u_{1}, v_{1}$, was obtained by applying one step of the block-byblock method suggested in Linz [6] to (1.2). The point $a$ was chosen to be a gridpoint of even index (cp. Table 6.1). The function $G(t+a)$ is evaluated according to (3.4): On $[0, a], K_{1}\left(t_{i}, u(s), v(s)\right)$ and $K_{2}\left(t_{i}, s, u(s), v(s)\right)$ are each approximated by piecewise quadratics and the necessary integrations are performed analytically. The second starting value for (3.5a) was obtained by using (3.6a) up to $a+h$. All resulting nonlinear equations were solved by Newton iteration. The numerical results for various $h$ are given in Table 6.1. The computations were done in double

TABLE 6.1

\begin{tabular}{|c|c|c|c|c|}
\hline$t$ & $\begin{array}{c}a=0.4 \\
h=0.1\end{array}$ & $\begin{array}{l}a=0.44 \\
h=0.05\end{array}$ & $\begin{array}{l}a=0.450 \\
h=0.025\end{array}$ & $\begin{array}{l}a=0.4750 \\
h=0.0125\end{array}$ \\
\hline 0.1 & $3.53715229 E-1$ & $3.53823239 E-1$ & $3.53818931 E-1$ & $3.53818448 E-1$ \\
0.2 & $4.88883032 E-1$ & $4.88809522 E-1$ & $4.88802420 E-1$ & $4.88801735 E-1$ \\
0.3 & $5.78790512 E-1$ & $5.78796717 E-1$ & $5.78790942 E-1$ & $5.78790440 E-1$ \\
0.4 & $6.42585689 E-1$ & $6.42542771 E-1$ & $6.42539428 E-1$ & $6.42539175 E-1$ \\
0.5 & $6.89183436 E-1$ & $6.89216386 E-1$ & $6.89214888 E-1$ & $6.89214802 E-1$ \\
0.6 & $7.24398475 E-1$ & $7.24383572 E-1$ & $7.24383127 E-1$ & $7.24383123 E-1$ \\
0.7 & $7.51593944 E-1$ & $7.51600536 E-1$ & $7.51600597 E-1$ & $7.51600626 E-1$ \\
0.8 & $7.73190081 E-1$ & $7.73187020 E-1$ & $7.73187284 E-1$ & $7.73187322 E-1$ \\
0.9 & $7.90688141 E-1$ & $7.90685735 E-1$ & $7.90686053 E-1$ & $7.90686089 E-1$ \\
1.0 & $8.05144191 E-1$ & $8.05144999 E-1$ & $8.05145307 E-1$ & $8.05145339 E-1$ \\
\hline
\end{tabular}


precision arithmetic on the IBM $360 / 50$ computer at the Australian National University.

Acknowledgment. This work was done while the authors were at the Computer Centre, The Australian National University, Canberra.

\section{REFERENCES}

[1] P. L. Chambre, Nonlinear heat transfer problem, J. Appl. Phys. 30 (1959), pp. 1683-1688.

[2] F. DE HoOG AND R. WEISs, On the solution of a Volterra integral equation with a weakly singular kernel, SIAM J. Math. Anal., 4 (1973), pp. 561-543.

[3] —-, Asymptotic expansions for product integration, Math. Comp., 27 (1973), pp. 295-306.

[4] J. B. Keller AND W. E. Olmstead, Temperature of a nonlinearly radiating semi-infinite solid, Quart. Appl. Math., 29 (1971-72), pp. 559-566.

[5] N. Levinson, A nonlinear Volterra equation arising in the theory of superfluidity, J. Math. Anal. Appl. 1 (1960), pp. 1-11.

[6] P. Linz, Numerical methods for Volterra integral equations with singular kernels, this Journal, 6 (1969), pp. 365-374.

[7] - The numerical solution of Volterra integral equations by finite differences, MRC Tech. Summ. Rep. 825, Mathematics Research Center, Univ. of Wisconsin, Madison 1967.

[8] J. N. LyNESS AND B. W. NinHAM, Numerical quadrature and asymptotic expansions, Math. Comp., 21 (1967), pp. 162-178.

[9] R. K. Miller AND A. Feldstein, Smoothness of solutions of Volterra integral equations with weakly singular kernels, SIAM J. Math. Anal., 2 (1971), pp. 242-258.

[10] B. NOBLE, Instability when solving Volterra integral equations of the second kind by multistep methods, Lecture Notes in Mathematics, 109, Springer-Verlag, New York, pp. 23-39. 